\title{
Novel Classifiers to Differentiate Focal and Macroreentrant Atrial Flutter Using 12-Lead Surface Electrocardiogram
}

\author{
Muhammad Usman Gul, Kushsairy Kadir, Muhammad Haziq Kamarul Azman \\ Universiti Kuala Lumpur, British Malaysian Institute, Gombak, Malaysia
}

\begin{abstract}
This article seeks to differentiate the mechanism of atrial flutter (AFL) between focal and macroreentrant from the surface ECG. It focuses on the hypothesis that the cycle length of visible consecutive $P$-waves ( $F$-waves) from the surface ECG can differentiate the mechanism (focal or macroreentry) of atrial flutter. Furthermore, early identification of the AFL mechanism from non-invasive techniques can improve the efficacy of invasive ablation. 12-lead surface electrocardiograms of AFL were collected from 48 patients whose mechanism was diagnosed by catheter ablation. Out of 48 recordings 42 were associated with a macroreentrant and remaining 6 were focal mechanism. The proposed model incorporates a wide range of features based on morphological and temporal properties of atrial activity. The wrapper technique has been used for the selection of best feature subsets and its performance is evaluated by using three different classifiers: Linear Discriminant Analysis (LDA), Logistic Regression (LOG), and Support Vector Machine (SVM). An over-sampling technique has been used to balance the dataset at seven different ratios. The best performance, at 5 times of minority (focal) dataset, has been achieved by LOG with maximum accuracy, specificity, and sensitivity of $92.41,99.26$, and 99.23 respectively.
\end{abstract}

\section{Introduction}

Atrial Flutter and Atrial Fibrillation (AFib) are the most common types of Supra-Ventricular Tachycardia (SVT). SVT is associated with serious heart-related complications including stroke and heart failure.

The underlying mechanism of AFL is focal (point) or macroreentrant (circuit). The most popular treatment for the AFL mechanism is Radio-frequency ablation. In such invasive ablation, during the electrophysiologic study, the analysis of intracardiac electrogram and pacing maneuvers is used to differentiate the mechanism of AFL [1]. But before starting an invasive procedure, the efficacy of the ablation procedure can be improved if we can identify the mechanism of AFL from the surface ECG.

There are various kinds of non-invasive procedures for identifying the mechanism of AFL. Currently, most of the researchers have proposed to improve the efficacy of the ablation procedure by BSPM (Body Surface Potential Mapping) [2]. In this way, a standard available ECG recording equipment could be used.

This paper aims to differentiate the focal from macroreentrant AFL by extracting features from 12-lead ECG during AFL. The variation in activation patterns which occur with focal and macroreentrant arrhythmia may be reflected in different delays between successive atrial activation as well as morphology of p-waves. To identify the hidden information behind the delay, this paper extracted time-based features as well as p-waves morphological features such as statistical values of peak-to-peak intervals, leads polarity, and maximum \& minimum distance between two peaks of atrial activation. As in reallife problems, the number of samples in one of the two available categories (focal or macroreentrant) was imbalanced. The majority categories are macroreentry over a minority focal AFL. To balance the dataset, Synthetic Minority Oversampling Technique (SMOTE) has been used to over-sample the minority class, with seven different ratios. Further, the performance of the three different classifiers has evaluated concerning maximum accuracy, sensitivity, and specificity.

\section{Material and Method}

\subsection{Data Description and Prepossessing}

A total of 61 ECGs were collected from a hospital. Each ECG is a 1-minute long recording. All 12-lead ECG recordings were taken during AFL ablation procedures at a sampling frequency of $2000 \mathrm{~Hz}$. A total of 13 files are excluded from the study because of excessive overlaps of $\mathrm{T}$ waves on $\mathrm{F}$ waves, due to a very low block ratio (less than $3: 1)$. In the remaining 48 recordings, 6 are related to focal atrial tachycardia and 42 are of macroreentrant atrial tachycardia.

To improve the signal-to-noise (SNR), an FIR filter has 


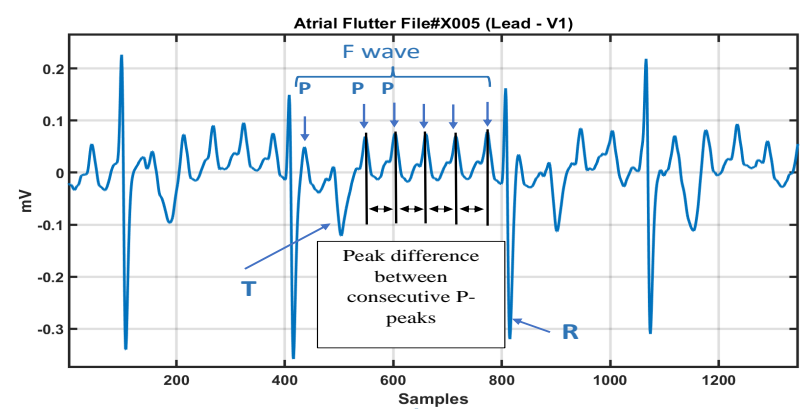

Figure 1. Proposed measures of peak-to-peak interval between consecutive $\mathrm{P}$-waves of extract F-waves in AFL

been used to eliminate the $50-\mathrm{Hz}$ power-line noises in ECG. Furthermore, using 0.5 to $60-\mathrm{Hz}$ Chebyshev typeII as a band-pass filter to mitigate the motion and highfrequency noises from the raw ECG.

\subsection{Detection and Segment of $F$-waves}

F-waves have information about the AFL mechanism and can differentiate the mechanism based on several types of features. Recent research has found two types of techniques for the extraction of $\mathrm{F}$-waves known as conventional and non-conventional techniques. The transform approach is playing an important role in non-conventional techniques. Wavelet transform (WT) [3]; addresses the drawback of Fourier transform (FT) and Short-time Fourier transforms (STFT) because it contains only frequency information and having constant window size respectively [4]. A Lot of different possible ways have been proposed for the extractions of $\mathrm{p}$-waves from the surface ECG, such as mathematical based morphology [5], wavelet-based transform [6], Hilbert-Huang [7] and algorithm based on machine learning [8]. In this paper, the wavelet-transform has been used to delineate the $\mathrm{R}$ and $\mathrm{T}$ waves [9] and eliminated them from the original ECG to obtain the F-waves. Fig 1 represented that how to calculate the peak difference between two consecutive P-waves to extract the F-wave from the surface ECG.

\subsection{Feature Extraction}

The collection of numeric features is derived from the raw ECG signals. These features are categorized into two main subsets, statistical and leads polarity of atrial activity. Statistical analysis is used to make a probabilistic determination of the variability in F-waves. For each recording, a series of F-to-F interval, corresponding to the duration between two consecutive $F$ waves measured from their peaks was obtained from multi-lead analysis of the surface ECG. From this interval series, nine statistical measures were adopted: mean, mode, median, standard deviation, vari- ance, skewness, kurtosis, maximum, and minimum distance between peaks of two successive p-wave. Three features (LII, LV1, LV6) are selected based on leads polarity from the surface ECG and one feature (LR) is on localization of the mechanism in atria. In total, 13 features are extracted from the ECG signal.

\subsection{Class Imbalance}

In a real-world applications, the class imbalance dataset is a common issue in machine learning. The class imbalance is predominant in this study, as 6 out of 48 records are associated with focal and remaining all are associated with macroreentrant atrial flutter.Various kind of techniques [10] have been proposed to offset the imbalance by re-sampling the dataset. Either down-sample the majority class or up-sample the minority class, this all depends on the task in hand.

The Synthetic Minority Oversampling Techniques (SMOTE) has been used to up-sample the minority classes [11]. After oversampling the dataset, the machine learner or classifier can now predict correctly within the decision regions for the minority class. However, it is suspect that a large oversampling may induce bias in the synthetic dataset, as well as performance. Moreover, in case it's too low, then the performance is not representative. There should be a nice rate that presents the best tradeoff. Seven different upsampling rates were used to balanced the minority dataset with the majority dataset. In details, over-sampled the minority dataset by introducing synthetic samples $1 \times, 2 \times, \ldots, 6 \times$ until it was balanced with the majority class.

\subsection{Classification and Feature Selection}

Each feature has a different level of information. Complexity and feature selection of the system are related to one another. Thus, feature selection is used to reduce the size of the problem, to decrease the system complexity as well as to improve the computational time. Additionally, feature selection allows us to evaluate the relevance of a feature and may also contribute to understanding how variability of AFL is manifested.

There are three common approaches (Wrapper, Filter, and Embedded) used for feature selection. In the wrapper approach, selected classifiers are part of the selection process. As compared to the other approaches, the filter method is the simplest one because it is usually based on the analytically compatible criteria. However, they do not take into account the classifier and its inherent complexity or strategy in learning.

Classifiers are used for training the data to discriminate the mechanism of AFL. The performance of these classifiers directly depends on the features of the dataset. Using 
Table 1. Feature Scoring using SMOTE with $5 \times$ Minority (42:30)

\begin{tabular}{|c|c|c|c|}
\hline Feature & LOG & LOG & SVM \\
\hline Mean & 0.62 & 0.62 & 0.69 \\
Median & 0.69 & $\mathbf{0 . 8 5}$ & $\mathbf{0 . 8 5}$ \\
Mode & 0.77 & 0.77 & 0.62 \\
Std & 0.54 & 0.54 & 0.77 \\
Var & 0.69 & 0.69 & 0.62 \\
Skew & $\mathbf{0 . 9 2}$ & $\mathbf{0 . 9 2}$ & $\mathbf{0 . 9 2}$ \\
Kurt & $\mathbf{0 . 9 2}$ & $\mathbf{0 . 9 2}$ & $\mathbf{0 . 9 2}$ \\
LII & 0.77 & 0.77 & 0.77 \\
L/R & 0.62 & 0.62 & 0.46 \\
LV1 & 0.46 & 0.46 & 0.69 \\
LV6 & 0.54 & 0.54 & 0.69 \\
PD $_{\text {min }}$ & 0.62 & 0.62 & 0.62 \\
PD $_{\text {max }}$ & 0.77 & 0.77 & 0.69 \\
\hline
\end{tabular}

wrapper technique to identify the features and their combinations to achieve the best performance of classifiers. In this technique, all possible combinations of features are tested. After evaluating the best performance of all feature combinations, determining at each length of combination the participation of a feature in the best subset (the one that gives the best performance) and assigning a score of 1 . We obtain the feature score. High scores indicate that these features are actively involved in the discrimination process and can help to clarify the factors that make it possible to discriminate against the focal point from the macroreentrant AFL. Three different types of classifiers have been used to evaluate their performance. The names of these classifiers are linear discriminate analysis (LDA), Logistic regression analysis (LOG), and linear support vector machine (SVM). Fig 2, is showing the performance of these classifiers concerning accuracy, specificity and sensitivity of all possible combinations.

\section{Results and discussion}

In Fig. 2 the mean values of accuracy, specificity and sensitivity of all three classifiers with respect to the feature subsets have been presented. The maximum accuracy, specificity and sensitivity of all three classifiers: LDA are $95.83 \%, 66 \%$ and $100 \%$; SVM are $100 \%, 50 \%$ and $100 \%$; and LOG have all are in $100 \%$. As observed, in the original data, all the classifiers performed with highest accuracy but have poor specificity except LOG classifier. Furthermore, the highest accuracy of the original dataset is due to highly imbalanced dataset. Therefore, the direction of the judgment boundary for the classifier trained with unbalanced data is biased towards the majority class and, as such, the predictive performance of the minority class is poor as compare to the majority. SMOTE technique has been used to overcome the data imbalance issue. Instead of balancing the dataset in a single step, minority class is increased gradually by an increment of $100 \%$ in each step. In this paper, seven
Table 2. Results of the AFL mechanism by using the Wilcoxon rank-sum test on synthetic dataset

\begin{tabular}{|c|c|c|c|c|}
\hline Feature & Macroreentry & Focal & p-value & $\mathbf{h}$ \\
\hline Mean & $431.91 \pm 111.51$ & $408.29 \pm 67.15$ & 0.023 & 1 \\
Median & $434.12 \pm 123.15$ & $470.93 \pm 25.40$ & 0.431 & 0 \\
Mode & $406.19 \pm 135.24$ & $265.1 \pm 168.79$ & 0.0005 & 1 \\
Std & $41.29 \pm 55.96$ & $127.76 \pm 68.19$ & $2 e^{-6}$ & 1 \\
Variance & $476.95 \pm 115.61$ & $237.92 \pm 173.77$ & $6 e^{-7}$ & 1 \\
Skewness & $-0.18 \pm 0.87$ & $-0.85 \pm 0.55$ & 0.0005 & 1 \\
Kurtosis & $3.71 \pm 2.10$ & $2.32 \pm 0.99$ & 0.0025 & 1 \\
LII & $-0.07 \pm 0.89$ & $0.167 \pm 0.59$ & 0.2244 & 0 \\
LR & $0.52 \pm 0.51$ & $0.63 \pm 0.49$ & 0.3617 & 0 \\
LV1 & $0.73 \pm 0.49$ & $0.3 \pm 0.70$ & 0.0035 & 1 \\
LV6 & $0.67 \pm 0.61$ & $0.3 \pm 0.60$ & 0.0046 & 1 \\
PD & $350.29 \pm 62.73$ & $219.3 \pm 142.55$ & 0.0005 & 1 \\
PD $_{\max }$ & $496.74 \pm 105.75$ & $527.87 \pm 30.91$ & 0.346 & 0 \\
\hline
\end{tabular}

steps have been applied on original minority class to balance the data. The performance of all of these steps has been evaluated and its results with respect to maximum accuracy, specificity and sensitivity have been shown in Fig. 2. For selecting the best one among the oversampled dataset, which has the best performance with respect to accuracy, specificity and sensitivity, the maximum averageaccuracy of each plot has been taken in account. Finally, the best performance at $5 \times$ of minority dataset (42:30) by LOG classifier have maximum average-accuracy, specificity, and sensitivity $92.41,99.26 \%$, and $99.23 \%$. Moreover, other classifiers SVM and LDA have also performed well with accuracy of $91.23 \% \& 89.53 \%$, specificity of $99.26 \% \& 97.98 \%$, and sensitivity of $92.30 \% \& 87.43 \%$ respectively.

The results of feature scoring for $5 \times$ SMOTE can be seen in Table 1. Where the maximum accuracy of the performance metric is taken into account. Several important features may be specified, arbitrarily defined by a score of $>0.80$. High scores suggest that these features are actively participating in the discrimination process and can help to explain the reasons that allows to discriminate against focal from macroreentrant AFL. Two features in all classifiers have highest involvement to differentiate focal from macroreentrant mechanism. These are features are skewness and kurtosis along with same score of 0.92 . These features with other relevant selected features have been highlighted in Table 1.

Applied Wilcoxon ranksum test for further validation of significant feature scores shown in Table 2. Finally, after the double test (wrapper approach \& Wilcoxon ranksum test), concludes that the two features, skewness, and kurtosis, play a major role to differentiate the AFL mechanism.

\section{Conclusion}

This study has utilized the 12-lead ECG and enhanced it further to understand the mechanics of focal atrial tachycardia. Results of our analysis have shown that variation 


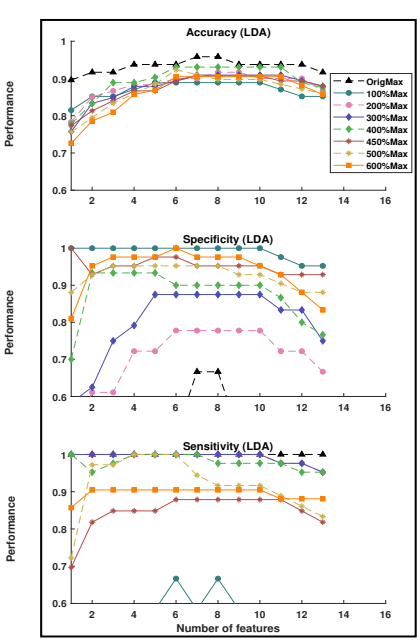

(a)

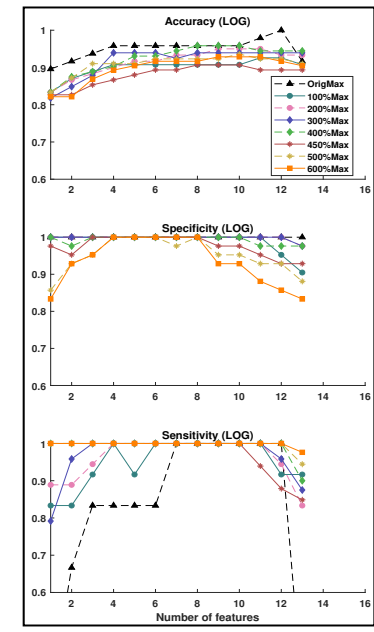

(b)

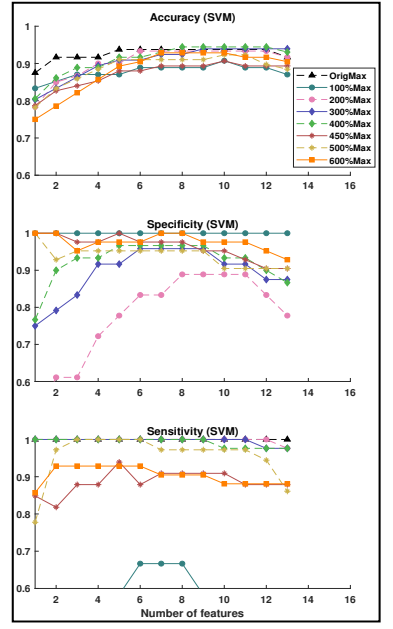

(c)

Figure 2. Performance evaluation of three classifiers: LDA, LOG and SVM with seven different oversampling rate up-to 100 to 600 percent. Accuracy, Specificity, and, Sensitivity of LDA, LOG, and, SVM are in (a), (b), and, (c) respectively. Whereas, $\mathrm{X}$-axis is the combination of features

of the successive intervals between atrial activities are related to the mechanism of atrial flutter. The cycle length of consecutive peaks of P-waves from the surface ECG has differentiated the focal from macroreentrant atrial flutter.

\section{Acknowledgements}

We thank Dr. D. G. Latcu from Centre Hospitalier Princesse Grace, Monaco for having provided us with the data for this study.

\section{References}

[1] MAINIGI SK, GREENSPAN AM. Contact impedance mapping: A new tool to differentiate focal from macroreentrant atrial arrhythmias. The Journal 2016;2271.

[2] MAINIGI SK, GREENSPAN AM. Utility of contact impedance mapping in differentiating the mechanism of focal atrial tachycardia. The Journal of Innovations in Cardiac Rhythm Management 2019;10:3642-3650.

[3] Lin HY, Liang SY, Ho YL, Lin YH, Ma HP. Discretewavelet-transform-based noise removal and feature extraction for ecg signals. Irbm 2014;35(6):351-361.

[4] Raj S, Ray KC. Ecg signal analysis using det-based dost and pso optimized svm. IEEE Transactions on instrumentation and measurement 2017;66(3):470-478.

[5] Yazdani S, Vesin JM. Extraction of qrs fiducial points from the ecg using adaptive mathematical morphology. Digital Signal Processing 2016;56:100-109.

[6] Yochum M, Renaud C, Jacquir S. Automatic detection of $\mathrm{p}$, qrs and t patterns in 12 leads ecg signal based on cwt. Biomedical Signal Processing and Control 2016;25:46-52.

[7] Li H, Wang X. Detection of electrocardiogram characteristic points using lifting wavelet transform and hilbert trans- form. Transactions of the Institute of Measurement and Control 2013;35(5):574-582.

[8] Kiranyaz S, Ince T, Gabbouj M. Real-time patientspecific ecg classification by 1-d convolutional neural networks. IEEE Transactions on Biomedical Engineering 2015;63(3):664-675.

[9] Gul MU, Kadir K, Azman HK, Iqbal S. Detection of r-peaks using single-scale wavelet transform. In 2019 13th International Conference on Mathematics, Actuarial Science, Computer Science and Statistics (MACS). IEEE, 2019; 15 .

[10] Seiffert C, Khoshgoftaar TM, Van Hulse J, Napolitano A. Rusboost: Improving classification performance when training data is skewed. In 2008 19th International Conference on Pattern Recognition. IEEE, 2008; 1-4.

[11] Chawla NV, Bowyer KW, Hall LO, Kegelmeyer WP. Smote: synthetic minority over-sampling technique. Journal of artificial intelligence research 2002;16:321-357.

Address for correspondence:

Muhammad Haziq Kamarul Azman

Universiti Kuala Lumpur, British Malaysian Institute

53100, Gombak, Selangor, Malaysia

mhaziq@unikl.edu.my 